\title{
Evaluation of the Quality of Expanded Program on Immunization Service Delivery in Primary Health Care Institutions of Jigjiga Zone Somali Region, Eastern Ethiopia
}

\author{
Ali Abdi Salah ${ }^{1}$, Nega Baraki², Gudina Egata², Wanzahun Godana ${ }^{3, *}$ \\ ${ }^{1}$ Somili Region Health Bureau, EPI coordinator, Jigjga, EasternEthiopia \\ ${ }^{2}$ School of Public Health, College Health and Medical Sciences, HaramayaUniversity, Harar, Ethiopia \\ ${ }^{3}$ Department of Public Health, College of Medicine and Health Sciences, Arba Minch University, Arba Minch, Ethiopia \\ Email address: \\ Wanzanati2011@gmail.com (W. Godana)
}

\section{To cite this article:}

Ali Abdi Salah, NegaBaraki, GudinaEgata, Wanzahun Godana. Evaluation of the Quality of Expanded Program on Immunization Service Delivery in Primary Health Care Institutions of Jigjiga Zone Somali Region, Eastern Ethiopia. European Journal of Preventive Medicine. Vol. 3, No. 4, 2015, pp. 117-123. doi: 10.11648/j.ejpm.20150304.14

\begin{abstract}
Introduction: quality is an integral part in EPI. Being pastoralist life style, poor infrastructure was considered the main reasons behind low coverage in this region. Therefore, the main aim of this study was to assess the quality of EPI service delivery in primary health care institutions of jigjiga zone Somali of region. Method: institution based cross-sectional study was carried out to assess the status of EPI service in PHCU of Jijiga zone, Somali region, from December 2011 to March 2012. Institutions were selected based on simple random sampling technique using mixed approaches. Results: This study has described the characteristics of quality of EPI service input, service process and service outcome main finding disclosed by this study, showed that almost the service is accessible to the care takers, most of the institution visited have adequate logistics and supplies and the service providers claiming, more than half of the mothers were satisfied with EPI services. The overall satisfaction in this study was $53.3 \%$. Out of 422 study participants, 405 (96\%) of the mothers/care takers were satisfied with the reception of the health workers. Conclusion: the study showed that half of the mothers yet not satisfied with the quality of the services. Therefore, to improve client satisfaction, waiting time should be kept as short as possible, in-service training/communication skill, outreach allowance should be considered for the service providers should be concerned woreda health offices, and further study in the area has to be made to dig out more factors affecting the service utilization of EPI.
\end{abstract}

Keywords: Quality, Pastoralist Community, Evaluation, Ethiopia

\section{Introduction}

Due to EPI, he lives of millions of children have been saved, millions have got the chance of a longer healthier life (1). Immunization service is the most powerful of all preventive health measures for children and it is central to human rights and poverty alleviation. (2). The ultimate goal of immunization programs is to reduce the incidence of vaccine preventable diseases (VPD) by children through attaining high levels of coverage with potent vaccines administered at the appropriate ages in the right intervals between doses for multiple dose vaccines (3). All countries have established national immunization programs, and in most developing countries, children under five years old are immunized with the standard WHO-recommended vaccines that protect against currently, eight diseases-tuberculosis, diphtheria, tetanus (including neonatal tetanus through immunization of mothers), Pertussis, polio, measles, hepatitis B, and $\mathrm{H}$. influenza type b. (4).

The Expanded Program on Immunization was launched in Ethiopia in 1980, to achieve universal child immunization by the year 1990. However, that remained unmet today. WHO/AFRO estimated that about five million children were un-immunized for DPT3 in 2007. Thus, the challenge to meet this goal cannot be ensured without considering the quality aspects of the program. So as to meet the immunization targets, quality of immunization service delivery is the suitability for providing the program in reliable manner, which is indicated by its components such as information and education, vaccine storage and handling and record 
keeping.(5).

The routine immunization coverage in Ethiopia has never reached the targeted figures and planned objectives. Sustainable improvements in the service delivery are needed in order to protect the Ethiopian children from unnecessary sufferings and deaths (6)

Similarly, the EDHS 2011 report, the coverage of EPI in Somali Region was low among the surveyed children on this region showed that only $16.6 \%$ of them were fully immunized while $35.4 \%$ were unimmunized and these figures are two times lower than the similar figures from other regions (7). The main reasons behind this very low coverage are postural life style, programmatic level to infrastructure conditions of the region. Others like little commitment in all levels, lack resource allocation, manpower and shortage of functional health facilities were also mentioned (2).

The immunization program in Ethiopia had crippled by limited resources and minimal capacity, it has little coverage $(52.5 \%)$, poor injection safety, and a deficient cold chain set up. (8). And mostly evaluations were focused on coverage related factors, but not to other dimensions, for example, the quality of immunization services provision in terms of from structure (resources), process (technique/knowledge) and outcome (satisfaction, coverage, etc) levels. The quality of EPI service has to be seen from the dimension of cold chain management, availability of logistics, and safety of injections and provider and client interactions.

Quality of health care is defined as having at least three dimensions. Which include structure, process and outcome (9). Structural quality refers to stable material characteristics (infrastructure, tools, and technology) and the resources of the organizations that provide care and the financing of care (levels of funding, staffing, payment schemes, and incentives). There is also input problems including disposable syringes and needles; refrigerators and the like(10, 11).

Adequacy of human resource although higher technology or a more pleasant environment may be conducive to better-quality care, the evidence indicates only a weak link betweensuch structural elements and better health outcomes (10).Many evaluations have revealed shortages in medical staff, medications and other important supplies, and facilities, but material measures of structure, perhaps surprisingly, are not causally related to better health outcomes (12).

In this study, attempt was made to measure the dimensions of quality of service outcome;input and the process. (13). Client satisfaction is an integral component of health service. The effectiveness of health care is determined to some degree by consumer's satisfaction with service provided and its quality the missing link $(1,14)$. The study conducted in rural Bangladesh showed that the most powerful predictor for client satisfaction was provider behaviour, especially respect, politeness and reduction in waiting time (15). So this study tried to assess the services based on the given components of quality. $(10,16)$.

\section{Methods and Material}

Study Area and Period: The study was conducted in Somali Region, Jigjiga zone from January to March, 2012.According to the population projections (CSA) made for 2010, the population of the region was $4,920,609$.Only $16 \%$. Live in town, while the rest are pastoralist and agro-pastoralist, the average house hold size is 6.6 and the average population density is 13 persons per square $\mathrm{km}$.

Study Design: institution based cross sectional study design was used.

Source Population:-

All governmental Primary health care institutions, all mothers with a child less than two years of age, who are visiting the health institution, and all immunization service providers in jigjiga zone

Study Population:

- A Random sample of selected health centres and health posts and all care givers and mothers/care takers who are visiting health institution during data collection in Jigjiga zone.

\subsection{Inclusion and Exclusion Criteria}

Inclusion criteria:-Health care providers who were assigned in the selected institutions to deliver EPI service during the time of data collection and mothers /caretakers who had children less than two years age and visited the selected health institutions during the study period.

Exclusion criteria: All non functional primary health care institutions and mothers who brought their children among the selected health institution for other purposes were not included in this study.

Sample size: the sample size was calculated assuming (p), proportion of clients satisfied with service provider interaction, $50 \%, 5 \%$ marginal error (d) and confidence interval of $95 \%$.and by assuming $20 \%$ non-response rate the sample was 461 .

\section{Sampling technique}

Firstly, the list of health centres and health posts were obtained from the Regional Health bureau. Six health centres and 12 health posts randomly were selected from the three levels (2HCs and 4 HPs from each level). The sample size was allocated to the randomly selected health institutions, based on their Penta 3 coverage. The average number of mothers/caregivers who come for EPI services were calculated and the number of interviewees per one day were determined.

\subsection{Data Collection and Management}

Quantitative data were gathered from clients, health institutions and health workers providing the service. Checklist developed to assess the health institution resources, the cold chain management, injection safety service delivery and other EPI supplies were observed including their functionality at each service delivery point. In-depth interview were done with one EPI coordinator per district and health extension workers per selected health institution, using interviewer administered a structured questionnaire to assess 
the knowledge of EPI services they provide. Mothers/care givers with children who came to the EPI services were interviewed at exit by using structured questionnaire.

\subsection{Study Variables}

Dependent: - EPI program implementation status

Independent variables:-Education status ofparents, Religion, Marital status, Occupation, Age, Income, Service provider's behaviour, Access to health service, previous history of problem after taking vaccination, Waiting time and discussion with health worker, availability of equipments and supplies, adequate human power,cold chain management, safe injection practices and mothers mother knowledge on immunization services

\subsection{Data Processing and Analysis}

Data entry, cleaning and analysis were made using SPSS Version 16 for windows statistical program. Association and relationships were computed using the bivariate and multivariate analysis techniques. The significance of associations was presented by p-values, and the $95 \%$ confidence interval of the adjusted odds ratios (AOR). On the other hand qualitative data wereanalyzed using theme.

\subsection{Data Quality Control}

The quality of data was ensured through training of data collectors and pretesting of the questionnaire. Supervisors and the principal investigator made frequent checks on the data collection process to ensure the completeness and consistency of the gathered information.

\subsection{Ethical Considerations}

Ethical clearance was secured from the Institutional Review Board of Haramaya University, College of Health and Medical Sciences IRERC. Then formal letter of cooperation were obtained from all concerned bodies at all levels. Informed verbal consentwas assured. The full information about the study was provided to the participants through a participant information sheet and additionally, participants were informed that they have a full right to refuse or discontinue participating.

\section{Results}

Out of the total of 461 mothers/caretakers, 422(91.5\%) responded, but all eighteen health institutions were observed as well EPI service providers interviewed.

\subsection{Socio-Demographic Characteristics of Study Population}

The highest proportion of mothers /caretakers 138 (32.7\%) were within the age group of 20-25 years, whereas age of their child $160(37.9 \%)$ were in the range 6-11month. Majority of the respondents $376(89.1 \%)$ were married. Regarding occupation of the interviewee house wife were predominant 309(73.2\%) (Table 1).
Table 1. Socio-demographic Characteristics of the mothers/care takers visited in PHC institutions of jigjiga zone, Somali Region. March 2012(N = 422).

\begin{tabular}{|c|c|c|c|}
\hline S.no & VARIABLES & Frequency & Percent \% \\
\hline \multirow{7}{*}{1} & Age of the mothers in years & & \\
\hline & $15-19$ & 46 & 10.9 \\
\hline & $20-25$ & 138 & 32.7 \\
\hline & $26-30$ & 111 & 26.3 \\
\hline & $31-35$ & 91 & 21.6 \\
\hline & $36-40$ & 29 & 6.9 \\
\hline & $41-45$ & 7 & 1.7 \\
\hline \multirow{4}{*}{2} & Age of the child in months & & \\
\hline & 0 - less than 6 months & 110 & 26.1 \\
\hline & $6-11$ months & 160 & 37.9 \\
\hline & 12 months and above & 152 & 36.0 \\
\hline \multirow{4}{*}{3} & Ethnic group & & \\
\hline & Somali & 359 & 85.1 \\
\hline & Oromo & 33 & 7.8 \\
\hline & Others (Harari,Amhara & 30 & 7.1 \\
\hline \multirow{3}{*}{4} & Religion & & \\
\hline & Muslim & 399 & 94.5 \\
\hline & $\begin{array}{l}\text { Others(Orthodox, Protestant, } \\
\text { catholic }\end{array}$ & 23 & 5.5 \\
\hline \multirow{6}{*}{5} & Education level & & \\
\hline & $\begin{array}{l}\text { Cannot read and Write(informal } \\
\text { education) }\end{array}$ & 203 & 48.1 \\
\hline & Rea and Write & 96 & 22.7 \\
\hline & Grade1-6 & 84 & 19.9 \\
\hline & Grade $7-12$ & 30 & 7.1 \\
\hline & Grade 12 and above & 11 & 2.6 \\
\hline \multirow{4}{*}{6} & Marital status & & \\
\hline & Married & 376 & 89.1 \\
\hline & Single/never married & 12 & 2.8 \\
\hline & Divorced and Widowed & 34 & 8.1 \\
\hline \multirow{6}{*}{7} & Current occupation of the respon & & \\
\hline & Government employee & 28 & 6.6 \\
\hline & Private enterprise employee & 2 & 0.5 \\
\hline & Merchant & 67 & 15.9 \\
\hline & House wife & 309 & 73.2 \\
\hline & Others & 16 & 3.8 \\
\hline \multirow{7}{*}{8} & Average monthly income of the $h$ & ehold & \\
\hline & Less or equal to 50 birr & 4 & 0.9 \\
\hline & 50-200 Birr & 6 & 1.4 \\
\hline & 201-450 Birr & 13 & 3.1 \\
\hline & 451-800 Birr & 36 & 8.5 \\
\hline & $>800$ Birr & 113 & 26.8 \\
\hline & Don’t Know & 250 & 59.2 \\
\hline
\end{tabular}

\subsection{Issues Related to Structural Quality}

In the selected health institutions and eighteen health workers who manage the EPI service were interviewed, by 
profession $11(61.1 \%)$ were clinical nurse, $6(33.3 \%)$ health extension workers and $1(5.6 \%)$ were junior nurse. From the visited 18 health institutions $15(83.3 \%)$ were not had transport facilities for outreach activity.Nine $(50 \%)$ of the health facilities had vaccination monitoring chart and were filled correctly, six $(33.3 \%)$ monitored the dropout rate monthly, $\operatorname{six}(33.3 \%)$ were had vaccine wastage monitoring and all the health institutions had adequate table and chair.

All health facilities had sufficient amount of AD syringes, and don't reuse needle and syringe. Eleven health facilities had enough wicks and glasses, 13 had weight scale for baby and all vaccine types /antigens were available, in those health facilities that were not had all vaccine types /antigens, BCG were commonly the missing vaccine.

Three hundred eighty seven $(91.7 \%)$ of mothers said the service was convenient for them, when asked reasons made to be convenient for the service, highest proportion $244(62.9 \%)$ replied, opening hours, and $108(27.8 \%)$ the distance travelled. When assessed the time (minutes) it takes them to reach this facility from their home by foot, almost $288(68.2 \%)$ of mothers/care takers replied less than 30 minute, and, the average waiting timeto get the service, 295(69.9\%) responded less than 30 minutes. Almost more than ninety percent of the study subjects addressed, that no other health institutionor vaccination outreach post nearer to them, on the other hand out of the eighteen facilities assessed, only $3(16.7 \%)$ had transport facilities for outreach activity.

\subsection{Issues Related to Process Quality}

Regarding injection safety16 EPI service providerswere used one mixing syringe for one vial for diluting vaccines andall health facilities had sufficient amount ofAD syringes, all the service providers didn't reuse needle and syringe, sevenseparate needles from syringes after use, also four recap needles and all of them were used safety box. Seventeen health facilities had incinerator and all of them were properly utilized. 13 had updated immunization guideline, eight were used vaccine stock balance sheets, 17 had cold box, and all health facilities had vaccine carriers.

Among 197(46.7\%) clients, who, were not satisfied in the service, reasons they mentioned was included, long waiting time for the service, child not weighted, and lack of chairs for sitting/tables, child developed problems after vaccination and the skill of the service provider.

Although, sixteen health providers reported, they had adequate EPI equipments and vaccine supply, but seventeen had cold box, and all had vaccine carriers. Regarding their source of power supply $8(44.4 \%)$ had $24 \mathrm{hrs}$ electric supply, $3(16.7 \%)$ from generator, $6(33.3 \%)$ were kerosene and 1 $(5.6 \%)$ were used solar as a source of power.

Among the selected health facilities, only nine health facilities $(50 \%)$ had vaccination monitoring chart and were filled correctly, six $(33.3 \%)$ monitored the dropout rate monthly, six( $33.3 \%)$ were had vaccine wastage monitoring and all the health institutions had adequate table and chair.

Fourteen $(77.8 \%)$ of the health institutions had been supervised in the last quarter out of them, nine $(64.3 \%)$ got feedback. All service providers responded yes, about explaining the schedule and contraindication of vaccine to their clients, but $16(88.9 \%)$ of the service providers check the immunization status of the children daily.Fifteen (83.3\%) EPI service providers were recorded the refrigerators' temperature twice a day including weekends in the last month, but, during observation the current temperature recorded $17(94.4 \%)$ of them were in between +2 oC to +8 oC. All health facilities had sufficient ice packs in the freezing compartment, of which only in one health facility, unnecessary materials were found placed on the top of the refrigerator. But all the vaccines were stored in their right compartment, only two $(11.1 \%)$ health facilities were found to store in wrong position.In all health facilities, vaccine vials which passed the expiry dates and vaccine vials without labels were not found in their refrigerators. Again only four health facilities $(22.2 \%)$ were found $\mathrm{BCG}$ and measles, which diluted before six hours.

\subsection{Issues Related to Outcome Quality}

The overall satisfaction in this study was $53.3 \%$. Out of 422 study participants, 405 (96\%) of the mothers/care takers were satisfied with the reception of the health worker, of which, the highest proportion $282(69.6 \%)$ of the clients satisfied was information given to them, the way of greeting and concern of the clients problem showed by the health worker were almost equal figures.

Among the seventeen mothers/care takers who were not satisfied with the reception of the health worker, when asked their main reasons become dissatisfied, 12 (70.6\%) replied since health workers was in hurry, followed by three $(17.6 \%)$ replied the health worker didn't show any concern to my problem, didn't gave us information and was rude or impolite was same figures.

\subsection{Result of Logistic Regression}

In order to assess the relative importance of each predictor, socio-demographic variables were analyzed if there is an association with satisfaction of the mothers'/caretakers' on EPI services they received from the selected health institutions.

In the multiple logistic regressionanalysis age, discussion with the health workers about immunization, time to reach health facility, and Waiting time to get the service were found to be significant determinants of satisfaction of the mothers'/caretakers' on EPI services they received from the selected health institutions. In the bivariate analysis educational status was associated with satisfaction but in multiple logistic regression it was confounded by variables when controlled (Table 2). 
Table 2. Association between Predictors variable and satisfaction variables among clients.

\begin{tabular}{|c|c|c|c|c|c|}
\hline \multirow{2}{*}{ Predators } & \multirow{2}{*}{ Categories } & \multicolumn{2}{|c|}{ Satisfaction of service with age } & \multirow{2}{*}{$\mathrm{COR}=95 \% \mathrm{CI}$} & \multirow{2}{*}{$\mathrm{AOR}=95 \% \mathrm{CI}$} \\
\hline & & Yes & No & & \\
\hline \multirow{6}{*}{ Age in years } & $15-19$ & 29 & 17 & 1 & 1 \\
\hline & $20-25$ & 74 & 64 & $1.5(.74,2.9)$ & $1.425(.700,2.902)$ \\
\hline & $26-30$ & 46 & 65 & $* * 2.4(1.22,4.9)$ & $2.22(1.06,4.64)$ \\
\hline & $31-35$ & 51 & 40 & $1.4(.65,2.8)$ & $1.17(.547,2.48)$ \\
\hline & $36-40$ & 20 & 9 & $0.77(.29,2.01)$ & $.66(.24,1.84)$ \\
\hline & $41-45$ & 5 & 2 & $0.67(.119,3.910)$ & $.675(.11,4.11)$ \\
\hline \multirow{5}{*}{ Educational status } & Cannot read/Write & 99 & 104 & 1 & 1 \\
\hline & Rea and Write & 51 & 45 & $0.840(.517,1.366)$ & $.867(.519,1.448)$ \\
\hline & Grade1-6 & 53 & 31 & $0.557(.330, .938)$ & $.569(.323,1.002)$ \\
\hline & Grade $7-12$ & 16 & 14 & $0.833(.386,1.796)$ & $1.016(.449,2.297)$ \\
\hline & Grade $12+$ & 6 & 3 & $0.476(.116,1.955)$ & $.359(.083,1.562)$ \\
\hline \multirow{2}{*}{ Discussion with HW } & Yes & 187 & 140 & 1 & 1 \\
\hline & No & 38 & 57 & $2.004(1.258,3.191)$ & $2.057(1.265,3.347)$ \\
\hline \multirow{2}{*}{$\begin{array}{l}\text { Time to reach the } \\
\text { health facility }\end{array}$} & Less than 30 minutes & 139 & 149 & 1 & 1 \\
\hline & Greater than 30 minutes & 86 & 48 & $* * 0.521(.341, .794)$ & $.557(.359, .865)$ \\
\hline \multirow{2}{*}{$\begin{array}{l}\text { Waiting time to get } \\
\text { the service }\end{array}$} & Less than 30 minutes & 147 & 148 & 1 & 1 \\
\hline & Greater than 30 minutes & 78 & 49 & $* * 0.62(.408, .953)$ & $.576(.368, .899)$ \\
\hline
\end{tabular}

** Significant associations

Reference category, Model coding - Yes $=1$, and $\mathrm{No}=0$

- COR- crude odds ratio,-AOR-adjusted odds ratio.

\section{Discussion}

This study has described the characteristics of quality of EPI service input, service process and service outcome (with the exception of coverage and effectiveness) main finding showed that almost the service is accessible to the care takers, most of the institution have adequate logistics and supplies and the service providers claiming, they have been trained and believing trainings are adequate to perform for the service, but about half of the care takers are were found not satisfied with the health care providers and feeling the service is not convenient for them and never assessed the degree of clients satisfaction before by any of visited health institutions.

The study indicated that about $72.2 \%$ health care professional providing the services expressed, that they were trained. Majority of them, $66.6 \%$ were nurses. Again the study found that all health institutions have adequate supplies for performing the immunization service, this indicates the presence of adequate human resource both professionally and in terms of skill mix. Thecurrent finding supported by the studies conducted 2002, in Dhaka city of Bangladesh revealed that at each of the 13 clinics, among workers who are giving immunization all but $76.9 .1 \%$ stated that, they had received on job training for immunization as well (16).

Similarly study done within the country, west Gojam found similar figures $68 \%$ service providers were trained in peripheral level EPI training. Even though most of those who had the training in one or other acknowledged that the training was sufficient to enable them to perform their duties effectively (11). This shows that, the providers of immunization service are almost knowledgeable to give technically appropriate immunization service.

The access to immunization service was measured in terms of travel time from client's village to the health institution and transport availability for outreach among institution. Regarding this, about $89 \%$ of the health workers agreed that the EPI supplies for their respective health facilities are adequate. Besides this, $83 \%$ of the health facilities involved had no transport facilities for outreach services. Though $68 \%$ of the mothers/care takers reaching the health institution within or less than 30 minutes from their residence, such structural access may be particular, only to the study area than rest of thein general and in sampled health facilities in particular.

These findings are generally not agreeable with the results of EDHS, 2005, indicated Somali, Gambella and Afar, the pastoralist lifestyle of the people in these regions create an added constrain in providing access to healthcare and have different needs from sedentary rural communities in rest of the country $(18,19)$. The survey also added health posts located in one area might be inaccessible for communities two or three years later.

Toward, the service process, evaluation dimensions was included, the current status of cold chain, injection safety and waiting time of the clients for the service.All visited institution had adequate amount of injection materials and highest proportion of them of providers had good injection and waste management. Only $23 \%$ were recap needle after use.The research done Oromia zone showed similar result with this study that all the service providers didn't reuse needle and syringe, didn't separate needles from syringes after use, also they did not recap needles.

Although all mothers had good knowledge about the immunization services and their commonest sources got the information was health providers. Similar the study done in Jimma (20) and Oromia zone of Amhara region did not show 
not much differences (21).

Regardless of good awareness on EPI service by the interviewed mothers/care takers among the selected health institutions, when asked their level of satisfaction towards reception of EPI service and their providers points of view, $96.0 \%$ of the mothers/care takers were satisfied with the reception of the health worker, of which $69.6 \%$ were satisfied due to the information given to them by the health worker, the way of greeting and concern of the clients problem by the health workers.

Almost out of 422 clients assessed their level of satisfaction about immunization service they got from these health institutions, $53.3 \%$ of them were satisfied. The main reason for satisfaction was $30 \%$ because of short waiting time of the service with other reasons. Similar study done in Jimma town showed that $35.4 \%$ of mothers could reach the vaccination site within 15 minutes and $49.2 \%$ of them could reach 15 to 30 minutes (20). Again the study done in west Gojam also showed that $98 \%$ of the mothers were satisfied with the service. Here it is good to integrate quality with client satisfaction (23). Twenty three percent were by the timelines of the services and $53 \%$ were satisfied by the effectiveness of the vaccine (11), this is different from, the respective study in jigjiga zone.

In this study predictors of mothers' satisfaction were age, discussion with the health workers about immunization,time to reach health facility, and Waiting time to get the service were found to be significant determinants of satisfaction of the mothers'/caretakers' on EPI services they received from the selected health institutions.

Another study done in rural Bangladesh showed that the most powerful predictor for client satisfaction was provider behavior, especially his respect, politeness and reduction of the waiting time $(1,15)$. Other study done in north showa concluded that interpersonal communication, processes including perceived empathy, perceived technical competency, non-verbal communication and patient enablement significantly influence patient satisfaction (22)

\section{Conclusions}

This study tried to assess the quality of EPI service provision among the primary health care institutions of jigjigazone, Somali region. All visited health institution were providing a sort of immunization service to their clients, and have one focal person to provide immunization and claiming technically confident, also all the necessary supplies, including cold chain equipments, injection materials/others and electric power was almost adequate. Unfortunately, half of the mothers were not satisfied about the service, reasons addressed are, due to long travel time to come the health institution, long waiting time to get served, poor reception and interpersonal communication from health staffs was a main outstanding issues. Hence technically competent workers could possibly affect the perception of the client and about all of the variables identified as independent predictors of patient satisfaction in this study.

\section{Recommendations}

Health workers should treat their clients and respect friendly; Unnecessary long waiting time or steps should be removed; missed opportunity from vaccine stock out should be corrected.Further studies is necessary to dig out more other problems challenging the coverage. Monitoring and supervision of health facilities is key to improve service quality

\section{Authors' Contributions}

AA was involved in the conception, design, analysis, report writing. NB, GE and WGhad been involved in the design, analysis and interpretation of the data, and report writing. In addition, WG was also involved in manuscript writing and review. All authors have read and approved the final version of the manuscript.

\section{References}

[1] WHO, 2009 World Health Organization ensuring the quality of vaccine at country level: Guidelines for health staff WHO: 2009: $1-30$.

[2] Koumaré AD. Traore, F. et al. 2009. Evaluation of immunization coverage within the Expanded Program on Immunization in Kita Circle, Mali: a cross-sectional survey BMC International Health and Human Rights9(1):13.

[3] WHO, UNICEF, World Bank. State of the world's vaccines andimmunization, 3rd ed. Geneva, World Health Organization, 2009

[4] Ronveaux O. et al.The Immunization data audit. Verifying the quality and consistency of immunization monitoring systems, Bull world Health org. 2005, 83: 503-10

[5] Yemane B, 2008. Universal Childhood Immunization: a realistic yet not achieved goal, Ethiopian journal of Health Development; 22 (2)

[6] United Nations children fund (UNICEF), World health Organization (WHO); 2007. Quarterly technical bulletin for managers of immunization services and health professionals, vaccines and immunization, Issue No. 1 Vol. 1, accessed on December 15, 2011

[7] Ethiopia Demographic and Health Survey (EDHS), 2011. Preliminary Report Central statistical Agency Addis Ababa, Ethiopia Measure DHS, Icf Macro Calverton, Maryland, USA

[8] Abdosh B: The quality of hospital services in eastern Ethiopia: Patient's Perspective.Ethiop J Health Dev 2006, 20:199-200.

[9] Stephen J \& R. Paul, 2003 Introduction to health service the 5 thedition. Assessing and implementing quality of care 2005, 467-73.

[10] Peabody, W.John, M. Taguiwalo, Robalino, A. David and J. Frenk, 2004 Disease control priorities in developing countries: Improving the Quality of Care in Developing Countries.

[11] Teshome, T. Assessment of quality of service delivery in immunization in West Gojam. AAU (unpublished) 
[12] Berhane, y. Bekele, A. Tesfaye, F. A special issue on immunization (EPI) in Ethiopia: Acceptance coverage, and sustainability ENJ April 2004; 38 (supplement) 35-55.North showa

[13] Donabedian A. 1999. The quality of care how it can be measured? Archives of pathology and laboratory medicine

[14] Jalal-EddeenAbubakar Saleh, HarunaIsmailaAdamu, Adamu Ibrahim Ningi. NNT Elimination and the Nigeria's Health System: Where is the Missing Link. Science Journal of Public Health. Vol. 3, No. 3, 2015, pp. 417-422. doi: 10.11648/j.sjph.20150303.28.

[15] MedozaAldana J. etal client satisfaction and quality of health care in rural Bangladesh. Bull world Health org. 2005, 79 (6): $512-7$

[16] Yibeltal K etal, 2010. Quality of Expanded Program on Immunization In Primary Health Care Facilities In Wolaita Zone, South Ethiopia, 2010

[17] Hennery P. et al. a comprehensive assessment of quality of immunization service in one major area of Dhaka city Bangladesh, Tropical Medicine and international health. December 1998 3/1/:981-992.
[18] Ethiopia Demographic and Health Survey (EDHS), 2005. Central statistical Agency Addis Ababa, Ethiopia Measure Dhs, Icf Macro Calverton, Maryland, USA

[19] Kidane T. and Takie M. Factors influencing child immunization coverage in rural district of Ethiopia, Ethiopian Journal of health development 2000, 17(2):105-110.

[20] Lankester T. Setting up community health programmes. Practical manual for use in developing countries, 2nd ed., London, pp 153-165

[21] NugusseAshene, 2006.Assessment of Quality of Expanded Program on Immunization in Oromia Zone of Amhara Region, (unpublished theses) Addis Ababa University School of Graduates Studies, Addis Ababa

[22] Birhanu Z, Assefa T, Woldie M, Morankar S. Quality of vaccination services and social demand for vaccinations in Africa and Asia. BMC Health Services Research 2010, 10:78.

[23] YeshambelAgumasAmbelie, AmsaluFelekeDemssie, MeashoGbreslassieGebregziabher. Patients' Satisfaction and Associated Factors among Private Wing Patients at BahirdarFelegeHiwot Referral Hospital, North West Ethiopia. Science Journal of Public Health. Vol. 2, No. 5, 2014, pp. 417-423. doi: 10.11648/j.sjph.20140205.17 